\title{
Ocular Artifacts Removal from EEG Using EMD
}

\author{
David Looney ${ }^{1}$, Ling $\mathrm{Li}^{1}$, Tomasz. M. Rutkowski ${ }^{2}$, \\ Danilo P. Mandic ${ }^{1}$, and Andrzej Cichocki ${ }^{2}$ \\ 1 Communications and Signal Processing Group \\ Department of Electrical and Electronic Engineering, Imperial College, London, UK \\ \{david.looney06, ling.li206, d.mandic\}@imperial.ac.uk \\ http://www. commsp.ee.ic.ac.uk/ \\ 2 Advanced Brain Signal Processing Laboratory \\ Brain Science Institute RIKEN, Wako-shi, Saitama, Japan \\ \{tomek, cia\}@brain.riken.jp \\ http://www.bsp.brain.riken.jp/
}

\begin{abstract}
Electroencephalogram (EEG) provides a non-invasive way to analyze brain activity. Blinking and movement of the eyes causes a strong electrical activity that can contaminate EEG recordings, particularly around the forehead but also as far as in occipital areas. Removal of such ocular artifacts is a considerable signal processing problem, since those artifacts overlap in frequency domain with EEG. In this paper we propose a signal reconstruction method based on a time frequency analysis tool known as the Hilbert-Huang spectrum. We demonstrate how our reconstruction scheme can be successfully applied to contaminated EEG data for the purposes of removing unwanted ocular artefacts.
\end{abstract}

\section{Introduction}

EEG is becoming a very popular brain activity analysis tool due to the low cost and easy application. Longer EEG recording experiments usually suffer from strong artifacts coming from muscle activity of which ocular artifacts, often recorded as reference in from of electro-oculorgrams (EOG), are the most common. The problem of EOG interference removal cannot be simply filtered out with conventional methods due frequency overlapping between EOG and EEG. We propose an adaptive approach based on the empirical mode decomposition (EMD), first introduced in [1], which is a fully data driven method for decomposing mutilticomponent signals into a set of amplitude \& frequency modulated (AM/FM) components known as intrinsic mode functions (IMFs). The IMFs act as locally orthogonal "basis functions" for the data, unlike other signal decomposition techniques that map the signal space onto a space spanned by a predefined basis. EMD has become an established tool in analysing real world data such as nonlinear and non-stationary signals with a number of important applications in signal processing. By definition, the IMF is a function for which the number of extrema and the number of zero crossings differ by at most one, and the mean of the two envelopes associated with the local maxima and local 
minima is approximately zero. The decomposition of a signal $x(k)$ is given by

$$
x(k)=\sum_{i=1}^{N} c_{i}(k)+r(k)
$$

where $c_{i}(k)_{i=1}^{N}$ is the set of IMFs and $r(k)$ is the residual. The first IMF is obtained as follows [1]: (i) Let $\tilde{x}(k)=x(k)$; (ii) Identify all local maxima and minima of $\tilde{x}(k)$; (iii) Find an "envelope," $e_{\min }(k)$ that interpolates all local minima $\left(e_{\max }(k)\right.$ maxima respectively); (iv) Extract the "detail," $c(k)=x(k)-$ $(1 / 2)\left(e_{\min }(k)+e_{\max }(k)\right) ;(\mathrm{v})$ Let $\tilde{x}(k)=c(k)$ and go to step 2); repeat until $c(k)$ becomes an IMF. Once the first IMF is obtained, the procedure is applied to the residual $r(k)=x(k)-c(k)$ to obtain the second IMF. In this way, the procedure is applied recursively to obtain all the IMFs. By definition, the IMFs are monocomponent signals and their instantaneous frequency can therefore be determined as defined by the Hilbert spectrum. Combining the instantaneous frequencies and corresponding instantaneous amplitudes of the IMFs, a timefrequency distribution known as the Hilbert-Huang spectrum can be constructed.

\section{Hilbert-Huang Reconstruction}

We now consider a unique reconstruction method based on the Hilbert-Huang spectrum which we refer to as Hilbert-Huang $(\mathrm{HH})$ reconstruction. Given a signal $d(k)$, we propose to remove any unwanted frequency information and construct a signal, $\hat{d}(k)$, that retains only desired frequency characteristics from $d(k)$. This is achieved by first decomposing $d(k)$ into a set of $N$ IMFs, $c_{i}(k)$, and determining the instantaneous frequencies. $f_{i}(k)$ denotes the instantaneous frequency of the $i^{\text {th }}$ IMF at time instant $k$. Given the scenario where it is required to retain frequencies greater that $f_{\text {low }}$ and lower than $f_{\text {high }}$, we have the following

$$
\tilde{c_{i}}(k)= \begin{cases}c_{i}(k) & \text { if } f_{\text {low }}<f_{i}(k)<f_{\text {high }} \\ 0 & \text { otherwise }\end{cases}
$$

Essentially all values of $c_{i}(k)$ that do not fall within the desired frequency range are set to zero. We can construct $\tilde{d}(k)$ by summation of the IMF values that fall within the desired frequency range.

$$
\tilde{d}(k)=\sum_{i=1}^{N} \tilde{c}_{i}(k)
$$

However, $\tilde{d}(k)$ is not a suitable solution on its own. Often it is the case that the instantaneous frequency of a particular IMF may intermittently become located within the desired frequency range. This has the effect of introducing unwanted discontinuities in $\tilde{d}(k)$ as certain IMFs are sparsely included in the summation process described above (4). These discontinuities have the potential to undo the goal of this report as their existence can reintroduce spurious frequency 
components in the data. To cater for this problem, we propose to determine the best approximation to $\tilde{d}(k)$ using the block based solution presented in [2]. The approximation, $\hat{d}(k)$, is achieved by linear summation of the original set of IMFs and residue using an optimally defined $(N+1) \times 1$ weight vector, w.

$$
\hat{\mathbf{d}}=\mathbf{w}^{T} \mathbf{I}
$$

where $\{\cdot\}^{T}$ denotes the transpose of a vector and $\mathbf{I}$ is a matrix containing the original IMFs and the residue (5).

$$
\mathbf{I}=\left(\begin{array}{cccc}
c_{1}(1) & c_{1}(2) & \ldots & c_{1}(M) \\
c_{2}(1) & c_{2}(2) & \ldots & c_{2}(M) \\
\vdots & \vdots & & \vdots \\
c_{N}(1) & c_{N}(2) & \ldots & c_{N}(M) \\
r(1) & r(2) & \ldots & r(M)
\end{array}\right)
$$

Letting $\mathbf{D}=\mathbf{I I}^{T}$, we can determine the optimal weight vector by the following

$$
\mathbf{w}=\mathbf{D}^{-1} \mathbf{I} \tilde{\mathbf{d}} \text {. }
$$

\section{Results and Conclusions}

We applied the $\mathrm{HH}$ reconstruction algorithm described above to several sets of EEG data contaminated by ocular artefacts. Since ocular artefacts occupy the critical low frequency band of EEG in a range $0-13 \mathrm{~Hz}$ [3] we proposed the carefully designed algorithm to retain the EEG important information within the above mentioned range while removing the ocular artifacts. The EEG data used was sampled at a rate of $512 \mathrm{~Hz}$ and recorded from electrodes $F p 1, F p 2, C 6$, $C 5, O 1, O 2$ with ground placed over $C z$ and additional bipolar channels $v E O G$, $h E O G$ as in standard extended 10/20 EEG system. The results are displayed in Fig. 1, and Fig. 2 respectively. To demonstrate how the algorihtm retains EEG information within frequency range originally contaminated by EOG we present time-frequency representations in forms of Morlet spectrograms in Fig. 2.

\section{References}

1. Huang, N., Shen, Z., Long, S., Wu, M., Shih, H., Zheng, Q., Yen, N.C., Tung, C., Liu, H.: The empirical mode decomposition and the hilbert spectrum for nonlinear and non-stationary time series analysis. Proceedings of the Royal Society A: Mathematical, Physical and Engineering Sciences 454(1971) (March 1998) 903-995

2. Weng, B., Barner, K.E.: Optimal and bidirectional optimal empirical mode decomposition. In: Proceedings of the 2007 IEEE International Conference on Acoustics, Speech, and Signal Processing. Volume III., Honolulu, Hawaii, U.S.A. (April 15-20, 2007) 1501-1504

3. Gasser, T., Sroka, L., Mocks, J.: The transfer of EOG activity into the EEG for eyes open and closed. Electroencephalography and Clinical Neurophysiology 61(2) (1985) 181-193 



Fig. 1. The results of the proposed approach to remove EOG artifacts. The top panel presents the recorded EEG at $F p 1$ electrode with very strong EOG artifact which is also presented as $v E O G$ channel in second from the top panel. The third from the top panel presents estimated EOG interference. The resulting and cleaned EEG is depicted in bottom panel, where no remains of EOG artifacts can be spotted.
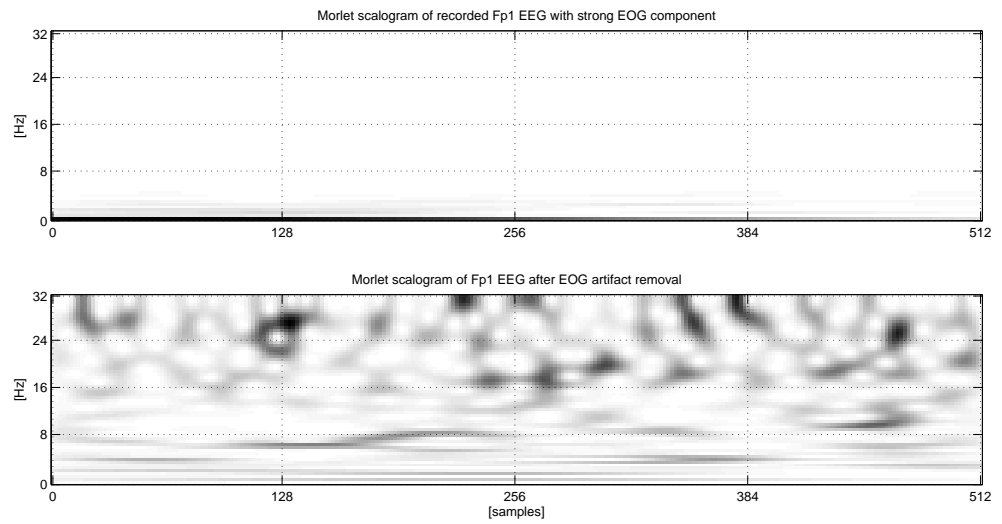

Fig. 2. Morlet spectrogram of the EOG contaminated EEG from the electrode $F p 1$ is shown in top panel. The very strong low frequency artifact from EOG is visible only around $\mathrm{OHz}$ and the rest of the spectrum is useless for analysis. The bottom panel presents the EOG removed EEG showing a very rich activity across the whole frequency range. It is important to note, that the proposed adaptive EOG removal approach does not damage low frequencies content, what usually happens with application of conventional filtering techniques. 